\title{
Challenges to Optimal Care for Orofacial Cleft Patients in Sub-Saharan Africa - The Example of Two Nigerian Tertiary Hospital
}

\author{
Akadiri Oladimeji Adeniyi*1, Amirize Ezekwe Ekwueme ${ }^{2}$ and Onah Ifeanyichukwu Igwilo ${ }^{3}$ \\ ${ }^{1}$ Department of Oral \& Maxillofacial Surgery, University of Port Harcourt, Nigeria \\ ${ }^{2}$ Department of Plastic Surgery, National Ortho paedic Hospital Enugu, Nigeria \\ ${ }^{3}$ Department of Surgery, University of Port Harcourt Teaching Hospital, Nigeria \\ Received: March 20, 2018; Published: April 06, 2018 \\ *Corresponding author: Amirize Ezekwe Ekwueme, Specialist Plastic Surgeon, University of Port Harcourt Teaching Hospital, Port Harcourt, Nigeria. \\ Formally Senior Registrar, Department of Plastic Surgery, National Orthopaedic Hospital Enugu, Enugu, Nigeria,Tel: 2348038659939; \\ Email: amirizee@yahoo.com
}

\section{Abstract}

Background: Complete and comprehensive management of a cleft patient goes beyond surgical repairs. However, certain challenges may limit total rehabilitation. This study intends to highlight specific challenges to optimal care for Orofacial cleft patients in the West African subregion and to suggest possible solution.

Methods: This is a cross sectional study carried out at two tertiary hospitals in Nigeria from January 2012 to December 2014. Cleft lip/palate patients were recruited from regular surgical outpatient clinics and during special surgical outreaches organized by the cleft teams of these hospitals. Consent was obtained from all participants (i.e. self or parental consent). In order to execute an inquiry into the challenges to effective care, a comprehensive preformed was drawn to elicit and record patients' and parents' bio-data, relevant history and clinical information as well as patients' perceived treatment needs and desired treatment outcomes. Separate inquiries were made to determine the professional group composition of the multidisciplinary cleft team in each hospital, available cleft care services in each hospital, and the treatment protocols observed by each cleft team. The data gathered were analyzed to determine the challenges to optimal care for the cleft patient.

Results: Two-hundred and twenty-eight (228) patients were recruited of whom 57 (25\%) were encountered during special surgical outreach programs. Patients' age range was between three weeks and thirty - six years. Observed challenges to optimal care are presented under four considerations namely; "Patient related", "Cleft team related", "Hospital related" and "Outcome related" Considerations. Broadly, the challenges include late presentation, high attrition rate, cleft teams' deficiencies in key specialty services (e.g. orthodontics, and speech therapy), and competing non cleft workload of the cleft team members.

Conclusion: Poor awareness, ignorance and financial difficulties accounted mostly for late presentation. Other barriers such as inadequacies of multidisciplinary cleft care services and required facilities in the hospitals were quite limiting. In order to mitigate the challenges, public re-orientation and patient education, infrastructural support, increased funding and training of essential personnel in the full range of skills for cleft care is imperative.

Keywords: Cleft Surgery; Non Surgical Therapy; Cleft Care Outcome; Cleft Awareness; Sub Saharan Africa

\section{Introduction}

There are various types of congenital craniofacial anomalies. Cleft lip and/or palate are the most common of these [1] In Nigeria, the prevalence rate of Orofacial clefts is 0.5 in 1,000 births [2] In conjunction with the cleft, there may be other associated malformations which vary in severity. Besides variable medical and dental complications associated with Orofacial cleft deformities, cleft patients and their parents suffer considerable psychological problems, stigmatization and social isolation; they also face huge financial burden required for corrective surgery and postsurgical rehabilitations [3]. Generally, the management of Orofacial cleft deformities is challenging and requires a well-coordinated multidisciplinary approach expected to start immediately after birth and continued until maturity or full rehabilitation [1]. However, in sub-Saharan Africa, late presentation is common and sometimes patients do not even present to the hospital at all. This is due to 
a number of psychological, socio-economic, infrastructural and human resource problems many of which have not been properly highlighted through scientific studies. This study therefore aims to highlight specific challenges to cleft care as it relates to patients, cleft team professionals and the health institutions in the West African sub-region and to suggest possible ways to overcome these challenges.

\section{Materials and Methods}

This cross sectional study was carried out at two tertiary hospitals where cleft care is provided in South-South and SouthEastern zones of Nigeria. These are University of Port Harcourt Teaching Hospital (UPTH) and National Orthopaedic Hospital, Enugu (NOHE) respectively. Other patients were captured during surgical outreaches within the zones but outside the respective hospitals. The study spanned the period from January 2012 to December 2014. Cleft team compositions and available services in each center were noted, Patients were involved after obtaining either parental (minors) or personal (adults) consent. Thereafter, patients' biodata and patterns of deformity were recorded. Presence or absence of syndromes was documented and necessary preoperative investigations were done. Interview and evaluation was conducted by members of the cleft team to determine diagnosis, individual patient's treatment needs, desires and expectations. Individual's experiences regarding factors affecting care obtainment and hindrances to follow up were recorded.

Factors affecting care delivery from the point of view of the multidisciplinary team of care providers were also recorded. Surgical repairs were carried out under general anaesthesia with end tracheal intubation executed by physician anaesthetists. Some adult primary lip repairs and lip revisions were carried out under field block and local infiltration of lidocaine with 1:100,000 Adrenaline. The standard protocol in both hospitals include cleft lip repair at age 3 - 4 months and Palatoplasty at 9-12 months. Patients that presented beyond this limit were charted as "late for presentation". Millard's rotation advancement flap and its various modifications was the most preferred technique for lip repair; Noodhorf vermilion flap technique was used occasionally where appropriate. For cleft palate repairs, von Langenbeck, intravelar veloplasty and Bardach 2-flap techniques were preferred with occasional use of Furlow's technque where lengthening was necessary. Follow up was planned for intervals of one week, 2 weekly, one monthly and then 3 monthly until further touch-ups or final discharge from outpatient care. Individual patient's compliance was monitored and the challenges/barriers to optimal management of each patient were critically examined and documented. Data collected were subjected to computerized statistical analysis using SPSS version 20.0 statistical software.

\section{Results}

The result is presented under four separate considerations namely; Patient related considerations, Cleft team related considerations, Hospital related considerations and Outcome related considerations.

\section{General Descriptive Statistics}

Two hundred and twenty-eight (228) patients were treated during the study period. Fifty-seven $(25.0 \%)$ of these were primarily seen during special outreach programmes carried out during the study period. There were 106 males and 122 females, giving a gender ratio of 1:1.2. Patients' age ranged between 3 weeks and 36 years with a mean age of $9.03 \pm$ SD8.30 years. Most patients $(63.6 \%)$ had first contact with the cleft team at ages above that which is recommended and adopted in the operative protocols of the cleft teams i.e. about 3 months for cleft lip, and 9-12 months for cleft palate. The remaining $23.4 \%$ came within the recommended time brackets. Majority had primary repairs while only $12.7 \%$ of the population came for secondary or revision procedures. Unilateral cleft lip constituted $40.3 \%$ of cases, bilateral cleft lip (9.2\%), isolated cleft palate (11.0\%), and combined cleft lip and palate $(26.8 \%)$. Of the secondary procedures, $7.0 \%$ were lip revisions, $0.4 \%$ alveolar bone grafting and $5.3 \%$ were closure of or nasal fistulae. There were syndromic associations including Treacher Collins, van der Woode and Pierre Robin Sequence in 1.7\%, 1.3\% and $0.9 \%$ of cases respectively and various forms of Tessier clefts were seen in $6.6 \%$ of patients (Table 1).

Table 1: Types of cleft cases and procedures done.

\begin{tabular}{|c|c|c|}
\hline & Cases for Primary procedures & Number of cases (\%) \\
\hline 1 & Unilateral cleft lip & $92(40.3 \%)$ \\
\hline 2 & Bilateral cleft lip & $21(9.2 \%)$ \\
\hline 3 & Isolated cleft palate & $25(11.0 \%)$ \\
\hline 4 & Combined cleft lip and palate & $61(26.8 \%)$ \\
\hline & Cases for secondary procedures & $16(7.0 \%)$ \\
\hline 1 & Lip revision & $12(5.3 \%)$ \\
\hline 2 & Oronasal fistulae & $1(0.4 \%)$ \\
\hline 3 & Alveolar bone grafting & \\
\hline 1 & Syndromic association/unusual & \\
\hline 2 & cleft types & $2(0.9 \%)$ \\
\hline 3 & Preacher Collins & $3(1.3 \%)$ \\
\hline 4 & van der Wobin Sequence & $15(6.6 \%)$ \\
\hline
\end{tabular}

\section{Patient Related Considerations}

Most patients (45.2\%) became aware of the free cleft treatment provided through various awareness and educational campaigns undertaken by the cleft teams. Awareness and referral were buttressed by cleft patients as well as their family and friends through spreading of information received to other people known to them (Figure 1). Observed challenges to optimal cleft care associated with patients are ignorance about the treatability of the condition, tendency to late presentation, poverty, non compliance with follow up schedule and non-completion of rehabilitation plan. Others are low threshold for satisfaction with aesthetic and functional outcome, associated systemic conditions and parental apathy. Majority dropped out of follow up within four weeks 
postoperative period while only sixty $(60 ; 26.3 \%)$ patients were available for follow up for up to 5 months (Figure 1).

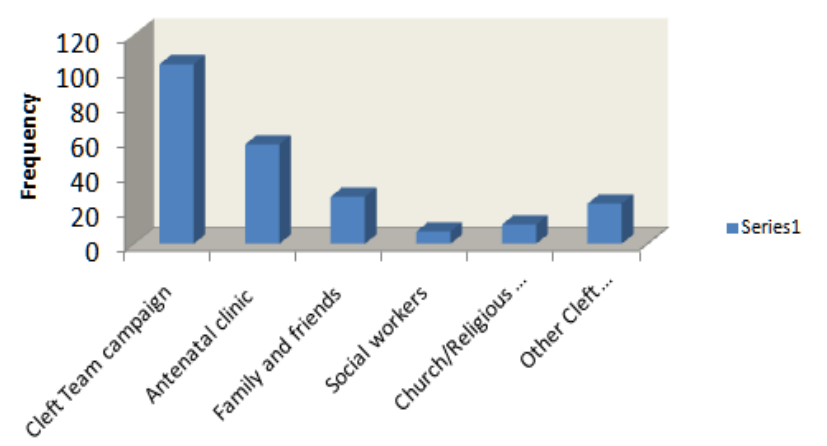

Figure 1: Patients source of information/referral to the cleft clinic/outreach.

\section{Cleft team Related Considerations}

The cleft team in the two hospitals differ slightly in compositions and services provided. While in one (UPTH), surgeons included plastic, oral and maxillofacial, and pediatric surgeons, in the other, only plastic surgeons were involved. Others members of the team in either hospital are physician anaesthetists, per operative and bedside nurses, speech therapists, orthodontists and social workers. One of the institutions (NOHE) also has dieticians and psychologists in the team. Orthodontics input at both centers was very minimal. Activities of the cleft teams included awareness and educational campaigns, media publicity and special outreaches, counselling/psychotherapy, corrective surgery and post-surgical follow up, speech therapy, dental and orthodontic rehabilitations and occasionally, nutritional support. The major barriers to optimal service from the cleft team are competing non cleft care related workloads of the participating specialists and inadequate funding to cater for non-surgical treatment needs of patients. Also noted was low passion on the part of non surgeon team members who felt "conscripted" to work with the team. Others were anaesthetic challenges such as difficult intubation and some untoward intraoperative events which occurred and accounted for some case postponement/ cancellations (Table 2).

\section{Hospital Related Considerations}

In both hospitals, cleft team was constituted by drawing specialists from the existing relevant departments who have additional non cleft related workloads. Cleft patients were seen in the general outpatient clinics among all other cases presenting to the hospital. A monthly dedicated cleft clinic was operated by one center. Patients' recruitment was augmented through special surgical outreach programs using either the same hospital or outside facilities for the surgery and immediate nursing care. Four such outreaches were undertaken during the study period and each lasted an average of four days. All laboratory services were provided by the respective hospitals at a cost borne by funding available to the cleft teams. Hospital related barriers to optimal care include challenges of case scheduling due to competing interests of other patients, inadequate bed spaces, case rescheduling/cancellation for various reasons (Table 2).

Table 2: Reasons for postponing or cancelling cases.

\begin{tabular}{|c|c|c|}
\hline & Reasons & Number of cases (\%) \\
\hline 1 & Cardiac anomaly & $6(2.6 \%)$ \\
\hline 2 & Underweight & $10(4.4 \%)$ \\
\hline 3 & Respiratory tract infection & $23(10.1 \%)$ \\
\hline 4 & Theater space unavailability & $49(21.5 \%)$ \\
\hline 5 & Difficult intubation & $2(0.9 \%)$ \\
\hline 6 & Transient apnea & $6(2.6 \%)$. \\
\hline 7 & Delayed recovery & $11(4.8 \%)$ \\
\hline 8 & Malignant hyperthermia & $2(0.9 \%)$ \\
\hline 9 & Industrial unrest & $23(10.1 \%)$ \\
\hline
\end{tabular}

\section{Outcome Related Considerations}

Long term outcome could not be sufficiently evaluated due to high attrition rate. However, complications such as wound dehiscence (3.9\%), vermilion notching (13.3\%), hypertrophic scars (16.8\%), persistent nasal deformities(31.8\%), and secondary palatal fistulae $(31.0 \%)$ (Figure 2) were observed within the period of follow up. The major challenge is that functional and aesthetic outcomes relating to patients' quality of life (such as speech, ear symptoms, feeding, and dental/orthodontic outlook) were not evaluated since most patients either did not begin or did not complete the treatments as planned at the time of loss to follow up (Figure 2).

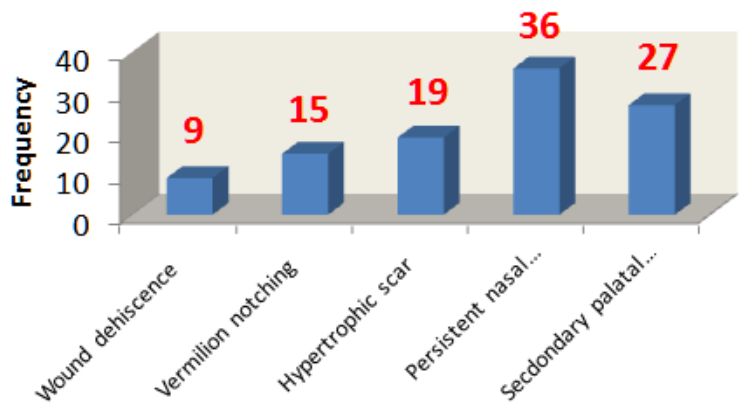

Figure 2: Suboptimal outcome requiring revision.

\section{Discussion}

Until the last two decades, the management of Orofacial cleft deformities was undertaken by relatively few surgeons in limited number of secondary and tertiary hospitals in Nigeria as well as most other West African countries [4]. Doubtless, the intervention of foreign donor organizations, especially the Smile Train, New York, USA, has significantly changed the face of cleft care and improved the fortune of individuals with congenital facial cleft deformities within the sub-region [5]. This intervention cuts across a wide area of needs, starting from funding of awareness campaigns and cost of 
surgical operations to increasing and improving local expertise in surgery, anaesthesia, nursing and speech therapy through training and capacity building. It also involves accreditation of several centers across the country with at least, the minimum infrastructure to guarantee safety. This humanitarian gesture has considerably reduced the major barriers and increased accessibility to cleft care. However, there are still some pertinent considerations that limit optimal care and complete rehabilitation of the cleft patient; most of these residual challenges are largely artificial.

In this study, we followed a cross section of Orofacial cleft patients managed under the funding program of Smile Train to identify specific challenges to achieving optimal correction, complete rehabilitation and social reintegration of the patients. The challenges were viewed in terms of patients related, cleft team related, hospital related and outcome related considerations. Of the two hundred and twenty eight patients studied, 25\% were encountered during outreaches; suggesting that still, a significant proportion of cleft patients would not voluntarily visit the hospital unless persuasive efforts and public education are brought to their localities to re-orientate and motivate them. Identifiable patient related barriers in the study still reveal elements of poverty, ignorance and low aspiration [6-8]. Prominent among these barriers is late presentation to the hospital. Reasons elicited for this behavior include lack of awareness that the condition is treatable or of a place where it could be treated, lack of financial resources to pay for treatment or to sustain the cost of travelling to the nearest place of care, unpleasant past hospital /surgery related experience and superstitious beliefs about the cause and the fate of the affected persons. Others reasons that have been expressed include fear of mortality occurring during surgical operation, lack of family and/ or social support [9] and unwillingness for treatment because of the parochial perception of economic benefits of physical disability achievable through street begging.

In our study, we encountered three parents who have untreated Orofacial cleft deformity as their children. It is distressing to note that one of the parents refused treatment despite the offer of free service probably for some of these sentimental reasons. This calls for intensive public health education, awareness campaign and provision of remedial education for parents and adult cleft patients. This task cannot be left for the cleft team, rather; it is a responsibility that must be embraced by public health experts, community health officers, government health policy makers and non-governmental agencies at all levels. In this study, majority of the patients became aware of the treatment opportunity for their condition through the community and media campaign undertaken by the cleft team. If this effort is driven at the primary levels by all, and especially community health workers and public health specialists, it will go a long way to help patient motivation and mobilization. Another prominent issue with the patients is that of non compliance with follow up schedule.

This is partly due to low threshold for satisfaction arising from initially repaired cleft lip and or even palate. Solving the obvious aesthetic disability seems to be all most patients desire, therefore, motivation for full rehabilitation such as speech therapy, orthodontic therapy and cosmetic touch ups is often lacking. This is unlike the experience of [10] in Texas, USA, who reported non-attrition of their patient population after years of follow up and they were able to achieve full rehabilitation of many. Another reason for defaulting include the high economic cost of transportation to the hospital which was not sustainable to the patients. Furthermore, some patients cited frustrations arising from some problems in the hospitals systems such as long waiting time, clinic schedule cancellation, postponement and rescheduling of treatment appointments sometimes as a result of industrial actions or infrastructural hiccups. Currently, the Smile Train is increasing the scope of support through provision of logistic costs to cover patients' transportation, rehabilitative treatments and other logistic costs to patients.

Hopefully, this will encourage follow up and facilitate evaluation of long term outcomes within our environment in the near future. The major barrier identified with the Cleft team is that of competing non cleft workload of personnel. As it stands today in most West African countries, we do not have teams dedicated exclusively to the care of cleft and other congenital anomalies. Hence, the cleft team is formed by drawing relevant specialists from various hospital departments whose primary workload is often already saturating. Sometimes, many of these staff (especially the support service staff) do not have original passion for cleft management and therefore sees the cleft workload as added burden. Even for those who are personally motivated, dividing their time between cleft patients and other patients could be a daunting task which adversely affect the scheduling and promptness of treatment for the cleft patients. Furthermore, the dearth of experts in areas such as speech therapy and orthodontics was underscored. While Smile Train has been able to train many surgeons and some anaesthetists, expertise in those fields such as orthodontics and speech therapy are comparably scarce in our environment.

Consequently, most centers do not have capable hands in these other important areas thereby de-optimizing outcome of cleft care. In the same vein, the activities of clinical psychologists, counsellors, social nurses, audiologist, geneticists, otolaryngologists, paediatric dentists, prosthodontists and physical anthropologists in our cleft teams have not been optimized, thus, limiting achievements in terms of social reintegration, self-discovery and personal esteem. It is important to have a comprehensive cleft team and run a dedicated multidisciplinary cleft clinic in order to articulate an encompassing treatment plan and treatment sequence which can be seamlessly executed within the same hospital. Fragmented care arising from inter unit or inter hospital referrals for needed services does not only lead to loss of patients' track and poor outcomes but it also prevent trainees and young specialists from learning the proper arts of structured treatment planning for the cleft patients [11]. Fear and anxieties also contribute to patients' discouragement.

The cleft team must be able to reduce intraoperative events and adverse experiences which may impact negatively on patients' perception of hospitals and the risks associated with surgical 
treatment. By cancelling cases due to adverse events such as difficult intubation and laryngospasms parents often feel threatened and sometimes do not return. It is particularly important to highlight the impact of hospital related barriers which tend to frustrate not just the patients, but also, members of the cleft team. The problems of underfunding and understaffing, logistic issues of epileptic essential services like power supply and central sterilization services, incessant industrial action by hospital staff all impacted negatively on the care of the cleft patient both in terms of treatment outcomes and promptness of treatment.

Patients/ parents who experience these hospitals related challenges tend to lose their motivation and this may account for perceived apathy to follow up and remedification of outcome related complications such as wound dehiscence, vermilion notching, unsightly scarring, nasal deformity and or nasal fistula. Therefore, it is equally important to minimize these outcome related complications through training and retraining of cleft team personnel to upgrade their respective skills. In order to mitigate many of these challenges, it is important to intensify the drive towards extensive and comprehensive public education with respect to Orofacial cleft deformities and formation of cleft clubs. In such clubs, individuals who have benefited from cleft repairs and rehabilitations can interact to encourage one another and help promote awareness among other affected individuals through their testimonies. This could help to increase antenatal screening and frequency of prenatal diagnosis, reduce late presentation and encourage early repair.

It could also reduce the psychosocial distress associated with cleft birth6 while also enhancing parents' adaptation 9 thereby improving the overall quality of life of both parents and patients. Also, for proper documentation of cleft birth, it is necessary to improve birth registration at local government levels in order to capture every child born in unorthodox birth centers and homes within the rural communities [2]. This way, early discovery of children born with facial cleft can be facilitated and patients can benefit from comprehensive neonatal assessments and pre-surgical managements including hearing assessment, visual assessment screening for other concomitant congenital abnormalities, pre-surgical orthopaedics, and nutritional support and where necessary, prompt referrals. Like the findings of [12] in Uganda most of our patients were seen outside the neonatal period. The adult palatal clefts were relatively wider with attendant challenges at Palatoplasty [13]. In addition, cleft teams may need to consider protocols that sustain parents' / patients' interest in the treatment course and/or reduce losses to follow up. For example, combined cleft lip and palate repairs have been suggested and performed in some cases [14].

Also, Palatoplasty preceding cheiloplasty has been advocated for some patients with cleft lip and palate so that patients do not drop out after the more aesthetically obvious cleft lip deformity has been fixed [15]. Above all, governments need to review their disposition towards congenital anomalies such as Orofacial deformities. There should be increased commitment to funding the care of indigent patients, policy review to promote societal consciousness about the condition and awareness of its treatability, and improvement of infrastructure and staff welfare at the hospitals to forestall unnecessary postponement, cancellations and incessant strikes. There is also the need to build capacity locally to augment available specialized skills required for comprehensive cleft management. Foreign help, though has had significant positive impact on cleft care in the West African sub-region, governments must reposition to sustain the gains because perpetual support from foreign agencies is not guaranteed. Much as the authors would have loved to compare the findings of this study with similar experiences reported in the literature, there are limited reports addressing the challenges of Orofacial cleft care from countries or sub-regions. Although, the authors are privy to know that situation in most West African country resemble the findings described in this study, a large scale multicenter international study is desirable to compare experiences across the world.

\section{Conclusion}

In summary, to mitigate the current challenges and optimize care for the cleft patients, extensive public education and reorientation, patient/parents education, infrastructural support, increased funding and training of essential personnel in the full range of skills for cleft care is imperative.

Acknowledgement: The authors wish to acknowledge the cooperation of members of the cleft team at UPTH and NOHE.

\section{References}

1. (1993) Parameters for the Evaluation and Treatment of Patients with Cleft Lip/Palate or Other Craniofacial Anomalies. American Cleft PalateCraniofacial Association. Cleft Palate-Craniofacial Journal 30 (Suppl 1): $1-16$.

2. Butali A, Adeyemo WL, Mossey PA, Olasoji HO, Onah II, et al. (2014) Prevalence of Orofacial Clefts in Nigeria. Cleft palate Craniofac Journal 51(3): 320-325.

3. Umweni AA, Ojo MA (2006) Orofacial Clefts: Team Approach to Management, $1^{\text {st }}$ Edn. BeninCity: Grafix Palace; Southern Nigeria, 2006: 3.

4. Efunkoya AA, Omeje KU, Amole IO, Osunde OD, Akpasa IO (2015) A review of cleft lip and palate management: Experience of a Nigerian Teaching Hospital. African Journal Paediatr Surgery 12(4): 257- 260.

5. Adeyemi TA (2015) Cleft lip and palate care in Nigeria: Current status of Orthodontic residents 'training in the management of children with cleft lip and palate. J Craniofac Surg 26(4): 1106-1108.

6. Onah II, Achor JU (2014) Psychiatric morbidity and quality of life among mothers of children with

Orofacial cleft disorders in Enugu: A pilot study. Nigerian J Plast Surgery 10(2): 10-14.

7. Opara KO, Olaitan PB, Onah II, Ogbonnaya IS (2008) cleft lip and palate repair: The experience from two West African sub-regional centers. J Plast Reconstruct Aesthete Surg 61: 879-882.

8. Fadeyibi IO, Fasawe AA, Jewo PI, Adeniyi AA, Ogunbanjo BO, et al. (2010) Nutritional and Hematological Parameters of Cleft Lip and/or Palate in Lagos, Nigeria, Nigerian Journal of Plastic Surgery 6(1): 16-22. 
9. Nakani M (2010) Negative Events Experienced by Mothers Raising Children with Cleft Lip and Palate. Kawasaki Journal of Medical Welfare 16(1): 43-49.

10. Wilhelmi BJ, Appelt EA, Hill L, Blackwell SJ (2001) Palatal Fistulas: Rare with the Two-Flap Palatoplasty Repair. Plast Reconstr Surg 107 (2): 315 318.

11. Bearn D, Mildinhall S, Murphy T, Murray JJ, Sell D, et al. (2001) Cleft lip and palate care in the United Kingdom: The Clinical Standards Advisory Group (CSAG) Study. Part 4: Outcome comparisons, training, and conclusions. Cleft Palate Craniofac Journal 38(1): 38-43.
12. Hodges SC, Hodges AM (2000) A protocol for safe anaesthesia for cleft lip and palate surgery in developing countries. Anaesthesia 55(5): 436441.

13. Amirize EE (2016) The relationship between cleft width and or nasal fistula. Nigerian J Plast Surg 12(1): 4-11.

14. Hodges AM (2010) Combined early Cleft lip and palate repair in children under 10 months - a series of 106 patients. Journal of Plastic, Reconstructive and Aesthetic Surgery 63(11): 1813-1819.

15. Agrawal K (2009) Cleft palate repair and variations. Indian J Plast Surg 42(3): 102-109.

\section{(C) (i) This work is licensed under Creative}

Submission Link: https://biomedres.us/submit-manuscript.php

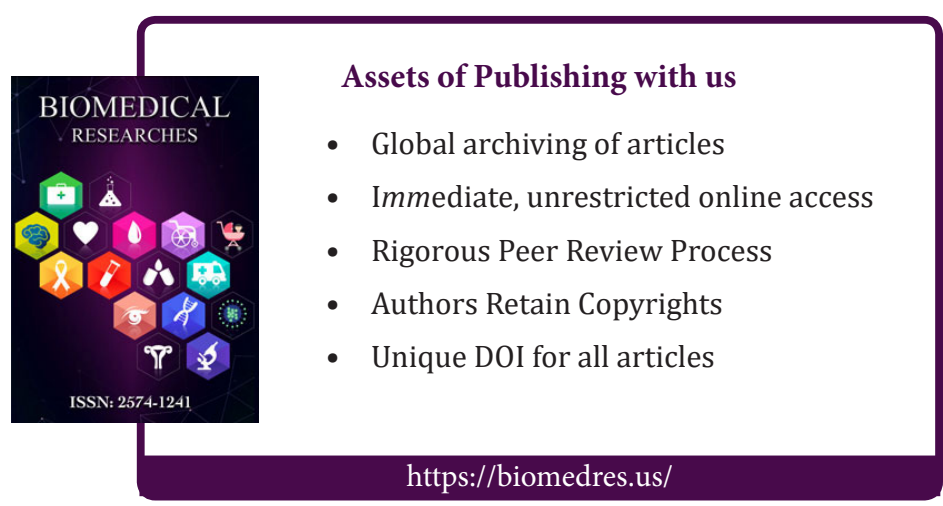

